\title{
"Unexplained" Delayed Death From Fungal Meningitis After Meningioma Resection
}

\author{
R. John Hurlbert, Juan M. Bilbao and William S. Tucker
}

\begin{abstract}
Background: Progressive deterioration and ensuing death following a neurosurgical procedure often represents a diagnostic challenge to the team responsible for patient care. Many, but not all, causes are treatable if a diagnosis is made early. Methods: A 69-year-old woman who died 6 weeks post-operatively following a meningioma resection is reported. An initial routine post-operative course became complicated by progressive neurological deterioration 3-4 weeks later. Despite extensive investigation she died 6 weeks post-operatively without a diagnosis. Results: Autopsy demonstrated extensive Candida meningitis. A review of the literature demonstrates this to be a reported complication in high risk patients, difficult to diagnose, but treatable when identified. Conclusions: Fungal meningitis should be high in the differential diagnosis in the post-operative patient with delayed, unexplained neurological deterioration, especially when associated with negative CSF cultures.
\end{abstract}

\begin{abstract}
RÉSUMÉ: Décès tardif inexpliqué d'un cas de méningite fongique après résection d'un méningiome. Introduction: La détérioration progressive, menant au décès du patient suite à une intervention neurochirurgicale, représente souvent un défi diagnostique pour l'équipe soignante. Plusieurs causes de ces décès sont traitables si le diagnostic est posé tôt, mais elles ne le sont pas toutes. Méthodes: Nous rapportons le cas d'une femme âgée de 69 ans qui est décédée 6 semaines après avoir subi la résection d'un méningiome. Les suites postopératoires, d'abord normales, se sont compliquées 3 à 4 semaines plus tard par une détérioration neurologique progressive. Malgré une investigation poussée, elle est décédée 6 semaines après l'opération sans qu'un diagnostic n'ait été posé. Résultats: L'autopsie a révélé qu'il s'agissait d'une méningite très étendue à Candida. Une revue de la littérature a montré que cette complication a été rapportée chez des patients à haut risque, qu'elle est difficile à diagnostiquer mais traitable quand elle est identifiée. Conclusions: La méningite fongique devrait être considérée en priorité dans le diagnostic différentiel du patient qui présente une détérioration neurologique tardive inexpliquée, surtout quand elle est associée à des cultures négatives du LCR.
\end{abstract}

Can. J. Neurol. Sci. 1995; 22: 239-243

CNS mycoses are an uncommon group of disorders with an often fatal outcome. They can be exceedingly difficult to diagnose, yet are treatable if identified early. Post-operative fungal meningitis is a reported complication following craniotomy. Several risk factors can predispose neurosurgical patients to this disease state. We describe the hospital course and autopsy findings of a patient with unrecognized Candida meningitis following craniotomy, and propose a high index of suspicion for avoiding delay in diagnosis in similar patients.

\section{CASE Report}

A 69-year-old right-handed, retired nursery school teacher presented with a five year history of unexplained falling. This event would occur two to three times per year and was characterized by a feeling of her "legs giving out." Her most recent episode was in the month prior to admission, occurring while descending a flight of stairs. She remembers waking at a local hospital with minor head abrasions. There had been no previous episodes of loss of consciousness. There was no previous history of seizures or dizziness. On close questioning, her son felt that the patient had exhibited mild confusion over the past 2-3 years, with increased dependence on family and friends. At the time of admission, she remained functionally independent, living on her own.
Her medical history included varicose vein stripping 20 years previously, and a detached retina with subsequent blindness in the right eye 10 years prior. She suffered from hypertension, benign positional tremor, and non-insulin dependent diabetes mellitus controlled by diet. Her medications included atenolol, enalapril maleate, and lorazepam. She had never smoked and did not consume alcohol. A positive family history was present for diabetes and hemophilia. Because of her most recent fall and resulting mild head injury, the patient underwent a $C T$ scan (Figure l) resulting in her referral to St. Michael's Hospital.

On admission, her blood pressure was 170/90. Her pulse, respirations, and temperature were normal. She was alert and oriented, but was extremely anxious with limited insight. Cranial nerve examination demonstrated complete blindness of the right eye with normal vision in the left. The left pupil reacted to direct light; the consensual reflex was present in the right eye. Cranial nerves $3-12$ were intact bilaterally. Muscle bulk, power, and tone were normal in all four extremities.

From the Division of Neurosurgery and the Department of Pathology, St. Michael's Hospital, Toronto.

RECEIVED NOVEMBER 15, 1994. ACCEPTED IN FINAL FORM MARCH 21, 1995.

Presented in part at the 29th Canadian Congress of Neurological Sciences meeting, St. John's, Newfoundland, June 1994.

Reprint requests to: Dr. W.S. Tucker, Chief of Neurosurgery, St, Michael's Hospital, 38 Shuter St., Toronto, Ontario, Canada M5B 1 A6 


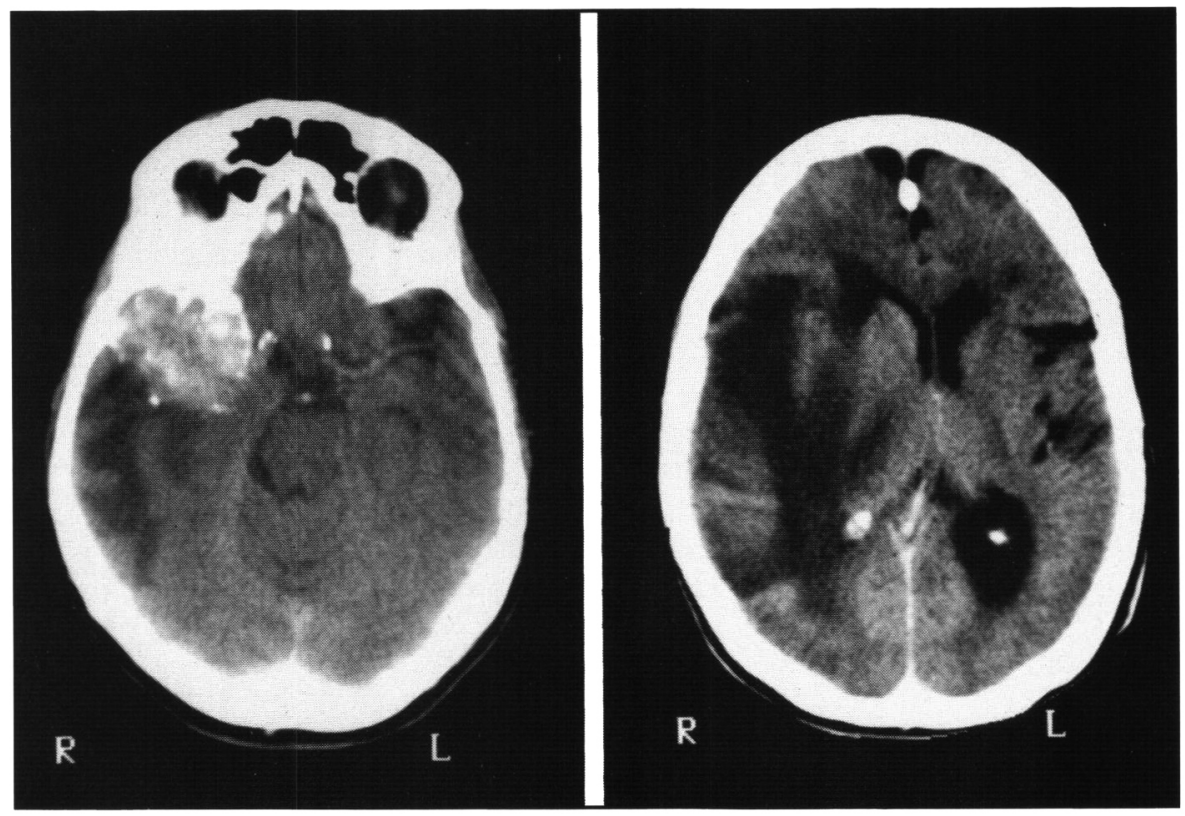

Figure 1: Enhanced CT scan at presentation. Left: An enhancing lesion with areas of calcification is seen in the middle fossa with associated edema and mass effect. Right: The edema is pronounced, extending through the parietal and into the occipital lobe.

Sensation was intact throughout. Her gait was slightly wide-based but coordination was normal. The deep tendon reflexes were symmetrically grade $2 / 4$ and both plantar responses were down-going. Examination of the other systems was unremarkable.

Shortly after admission, the patient underwent a right fronto-temporal craniotomy and gross total resection of a meningioma arising from the floor of the middle cranial fossa (petrous temporal bone). The tumor was of fibrous consistency, moderate vascularity, and had a well defined capsule. The dural base was cauterized completing a Simpson grade II resection. Histologically, the tumor demonstrated brain invasion and individual cell necrosis. However, mitosis were infrequent. The final diagnosis was atypical meningioma. Immediately post-operatively, the patient was confused to place, obeying commands, and had a mild left arm drift. Because of continuing confusion, a repeat CT scan was performed on the third post-operative day demonstrating persistent cerebral edema. Her dexamethasone was increased back to $4 \mathrm{mg}$. qid and she was fluid restricted because of a serum sodium of $132 \mathrm{mmol} / \mathrm{L}$ (normal 135-145). Over the next 4 days the patient improved clinically, becoming fully oriented with resolution of the left arm drift. She was discharged home 9 days post-operatively on a weaning dose of dexamethasone.

Two weeks later, while at home, her daughters noticed progressive confusion and inappropriateness. Within 48 hours, she was unable to ambulate and was taken to a nearby hospital. A CT scan demonstrated some persistent edema, but the midline shift had resolved. No evidence of residual tumor was present on the enhanced scan. The patient was transferred back to St. Michael's Hospital on atenolol, enalapril maleate, folic acid, phenytoin $300 \mathrm{mg}$ qhs, dexamethasone $4 \mathrm{mg}$ q6h, and ranitidine hydrochloride $150 \mathrm{mg}$ bid. Physical examination revealed a blood pressure of $160 / 90$, a pulse of 72 , normal respirations and an oral temperature of $36.9^{\circ} \mathrm{C}$. She was confused to place and time, was poorly cooperative, exhibited comprehension difficulties and a decreased attention span. Her GCS was 14. The remainder of the neurological examination was unchanged from her original admission. Although there was some discomfort to palpation over the craniotomy site, the wound was healing well and there was no evidence of nuchal rigidity. Pertinent laboratory investigations demonstrated a haemoglobin of $134, \mathrm{WBC} 7.9, \mathrm{Na}^{+} 125 \mathrm{mmol} / \mathrm{L}$, serum osmolality $262 \mathrm{mosm} / \mathrm{L}$, glucose $14.2 \mathrm{mmol} / \mathrm{L}$, and a dilantin level of $15 \mu \mathrm{mol} / \mathrm{L}(40-80)$. An EEG demonstrated generalized slowing of activity greater in the right hemisphere with right sided epileptogenic spikes. The patient was admitted with a tentative diagnosis of SIADH and seizure disorder. She was placed on a $1200 \mathrm{cc} / 24 \mathrm{hr}$ fluid restriction and her dilantin was increased to $200 \mathrm{mg}$ bid.

Over the next two days, she demonstrated a labile affect, crying for no apparent reason several times during the day. Three days after admission, she was more drowsy, not opening her eyes and obeying commands only intermittently $(\mathrm{GCS}=11)$. Her $\mathrm{Na}^{+}$was $126 \mathrm{mmol} / \mathrm{L}$ and her dilantin level was 40 . Demeclocycline was started. A repeat EEG showed reduced spike activity. Neurological consultation suggested the patient was suffering from periodic lateralizing epileptic discharges (PLEDS). She continued to deteriorate and by the eighth day of admission her GCS was 7 (eyes closed, no sounds, localizing pain). In addition, she began to show signs of right sided weakness. She remained afebrile and did not exhibit any signs of meningismus. At this time her $\mathrm{Na}^{+}$was $130 \mathrm{mmol} / \mathrm{L}$ and her dilantin level was $53 \mu \mathrm{mol} / \mathrm{L}$. A lumbar puncture was performed; the CSF was clear and colourless with $2 \mathrm{RBCs}$ and 150 WBCs ( $16 \%$ polymorphonuclear cells, $84 \%$ lymphocytes). The Gram stain was negative. CSF glucose was $5 \mathrm{mmol} / \mathrm{L}(60 \%$ serum glucose) and protein was $1.07 \mathrm{~g} / \mathrm{L}(0.15-0.40)$. Latex agglutination for Cryptococcal antigen was negative. On the ninth day of admission she lost her right corneal and gag reflexes, and developed bilateral Babinski responses. Her blood pressure was $140 / 90$, pulse 90 , respirations 30 , and temperature $37.9-38.7^{\circ} \mathrm{C}$. A repeat $\mathrm{CT}$ scan showed a large left fronto-parietal infarct and a right occipital infarct (Figure 2). Carotid dopplers and an echocardiogram were normal. Blood, urine and sputum cultures were negative. CSF cultures remained negative. Twelve days after admission her GCS was still 7. Consultation with the family resulted in withdrawal of active intervention. Fifteen days after admission (6 weeks post-operatively) the patient expired.

Permission was obtained for a complete autopsy. Superficial venous thrombosis was noted over the left fronto-parietal cortex with a large underlying hemorrhagic infarction. In addition, there was a large hemorrhagic infarction of the right occipital lobe and a smaller one in the right frontal lobe. A thick, patchy white exudate was centred over the right fronto-temporal region at the operative site, also infiltrating the base of the brain (including the Circle of Willis) and the brainstem. The exudate continued caudally, involving the leptomeninges of the spinal cord as well. Microscopically, focal arterial occlusion of the basilar perforators was apparent, with micro-infarction of the pons. A widespread, chronic granulomatous meningitis was present involving the brain, brainstem, and spinal cord. Budding yeast with hyphal forms, morphologically consistent with Candida, were prevalent throughout (Figure 3). The final diagnosis of Candida meningitis was based on histopathological 

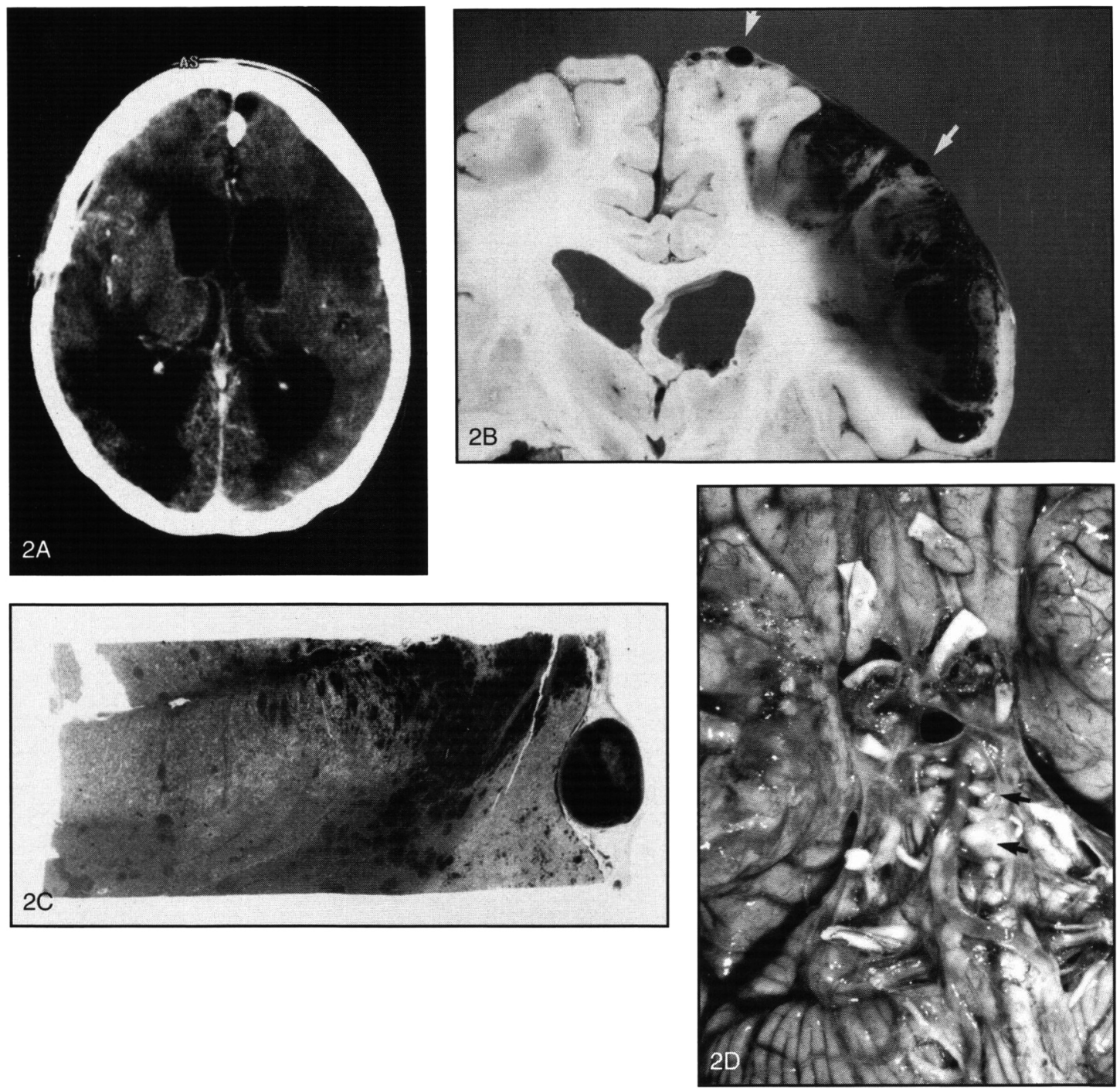

Figure 2: A. An enhanced CT scan performed 6 days prior to death demonstrates hydrocephalus, periventricular edema, and left frontal and right occipital infarcts. $\boldsymbol{B}$. Post-mortem examination of the brain demonstrates the hemorrhagic nature of the left frontal infarct. Superficial venous thrombosis is seen at the surface of the infarct and near the interhemispheric fissure (arrows). C. The whole mount preparation more clearly illustrates the superficial venous thrombosis at the cortical surface and the underlying hemorrhagic infarction. D. The basal surface of the brain shows focal thickening and opacification of the leptomeninges. In addition, nodular areas of granulation are seen along the interpeduncular cistern (arrows).

criteria alone; post-mortem cultures could not be obtained from the formalin fixed brain tissue. Ante-mortem fungal CSF cultures remained negative for 4 weeks after the lumbar puncture. General autopsy revealed no evidence of disseminated or focal extra-cranial Candidiasis.

\section{Discussion}

Fungal infections of the CNS are a rare group of disorders that typically affect the immunocompromised host. Cryptococcus neoformans is the most common pathogen producing meningitis in up to $11 \%$ of HIV infected patients. ${ }^{1}$ However, in non-AIDS patients, Candida albicans is the most prevalent pathogen at autopsy, isolated in $40-50 \%$ of patients dying from CNS mycoses. ${ }^{2.3}$ Interestingly, reports of CNS candidiasis are largely restricted to autopsy series and therefore may not reflect the true incidence of the disease. Nonetheless, Lipton et al., in presenting both clinical and autopsy material of patients with documented CNS Candida mycosis, reported only one of fourteen patients alive. ${ }^{3}$ Survival from the time of diagnosis ranged from 2 days to 2 months in the other patients. Even in cases of treated chronic Candida meningitis, the mortality rate is $33 \%{ }^{9}$ Thus the difficulty in making the diagnosis, and the subsequent poor outcome of this disease are underscored. 


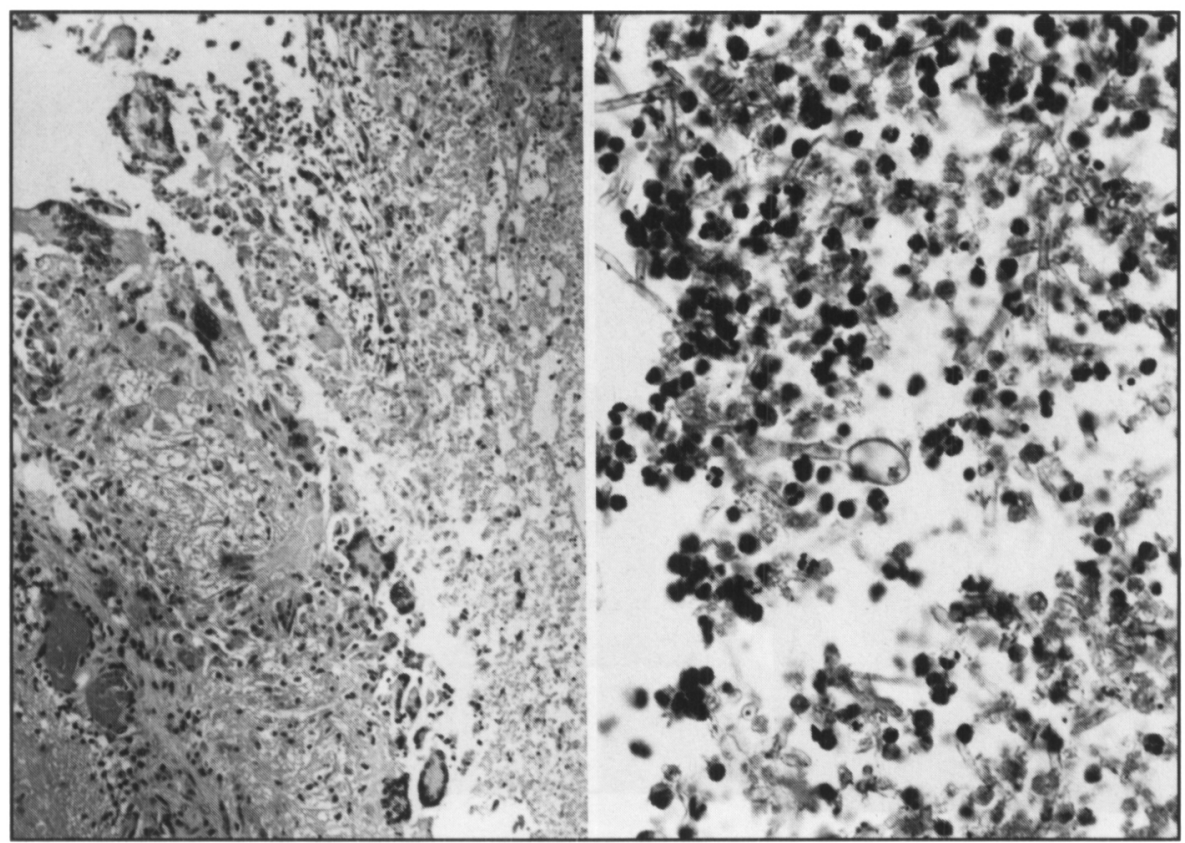

Figure 3: A. Under low power, microscopic inspection of the arachnoid and pia from the base of the brain reveals a florid acute and chronic granulomatous meningitis. Haematoxylin and eosin stain. B. High power examination demonstrates exuberant proliferation of a fungus demonstrating hyphal and yeast-like (budding) forms, typical of Candida. PAS stain.

Several risk factors are associated with Candida CNS infections (Table). Candida meningitis occurs most commonly in infants and the elderly, classically being associated with immunocompromise or broad spectrum antibiotic therapy. ${ }^{3,4}$ Fungal meningitis has also been reported in association with diabetes and i.v. drug abuse. ${ }^{5.6 .9}$ CNS Candidiasis has been reported in up to $80 \%$ of patients with systemic Candida infection. The most common portals of entry include the gastrointestinal, pulmonary, and urinary systems. ${ }^{3}$ Isolated CNS Candidiasis is much less common. ${ }^{5}$ Independent risk factors for CNS infection include previous surgery as well as systemic corticosteroids. ${ }^{7}$ Surgery has been found to specifically predispose to Candida infection as opposed to other fungal mycoses. In 1986, Fleischmann et al. found only 77 reported cases of Candida meningitis in the published literature. ${ }^{5}$ Of these, 6 were associated with CSF diversionary procedures and 2 were related to other neurosurgical interventions. More recently, another case associated with debulking of a glioblastoma has been reported. ${ }^{8}$

Meningeal signs, papilledema, and focal neurological deficits can be rare in Candida CNS mycoses. They were present in $<10 \%$ of patients dying from CNS candidiasis, whereas seizures were present in $15 \% .^{7}$ Meningismus was much more common in Cryptococcal infections (86\%). The antemortem diagnosis of Candida CNS mycoses is very difficult, being made in only three of $14(21 \%)$ cases in one autopsy series, and in 0 of 27 cases in another. ${ }^{3,7}$ In a recent review of selected patients with chronic Candidal meningitis, CSF smears were positive in only $17 \%$ of patients while cultures were positive in $44 \% .^{9}$ Demonstration of fungi in the CSF remains the gold standard for diagnosis, but as is apparent, has a high incidence of false negative results. Centrifugation and filtration of relatively large CSF volumes may improve the sensitivity of CSF examination.
As with a large proportion of the neurosurgical population, our patient had many of the risk factors associated with fungal CNS infections. She was elderly, on peri-operative systemic steroids, and had undergone a surgical procedure. In addition, our patient was diabetic. Although she was not on long-term antibiotics, she did receive peri-operative prophylaxis. She did not exhibit meningeal signs at any time and only developed a fever in the end-stage of the disease. Fungal studies of the CSF, including India Ink staining and cultures, were completely negative. In hindsight, the only clue to her underlying condition came indirectly from the results of the lumbar puncture, where the white count was moderately high with a relative lymphocytosis and elevated protein. However, at the time, these changes were felt to be non-specific and likely related to her relatively recent surgery. Had a diagnosis even been established by the onset of venous thrombosis and infarction, institution of antifungal therapy would doubtless have been too late. In retrospect, considering the patient's obvious deterioration, early lumbar puncture and a high degree of suspicion might have helped us make an antemortem diagnosis. In this case, confirmation would have required wound re-exploration and biopsy of the meninges.

Table: Risk Factors Associated with Candida CNS Infections.

$\begin{array}{ll}\text { 1. } & \text { Age (premature infants and the elderly) } \\ 2 . & \text { Immunocompromise } \\ 3 . & \text { Broad spectrum antibiotic therapy } \\ 4 . & \text { Systemic Candidiasis } \\ 5 . & \text { Systemic corticosteroids } \\ 6 . & \text { Surgery } \\ 7 . & \text { CSF diversion } \\ 8 . & \text { Diabetes } \\ 9 . & \text { I.V. Drug abuse }\end{array}$


The treatment for systemic infections due to Candida has classically been with the antifungal agent amphotericin B, which can also be given intrathecally. ${ }^{3}$ However, the drug is often associated with unpleasant side effects which can preclude long-term administration. For Candida infections of the CNS, parenteral amphotericin B and oral 5-fluorocytosine are felt to work synergistically to improve response rates. ${ }^{4,9,10}$ Resistance to 5-fluorocytosine alone is common. ${ }^{10} \mathrm{~A}$ combination of fluconazole and 5-fluorocytosine for a period of 8 months has been recently used to successfully treat Candida meningitis in a patient with amphotericin B intolerance. ${ }^{9}$

In summary, neurosurgical patients as a whole often possess more than one risk factor for the development of fungal meningitis. The clinical presentation is typically vague and insidious. Laboratory investigation is often helpful only in excluding other post-operative conditions; CSF culture results are routinely negative. In a post-operative patient with unexplained delayed neurological deterioration, fungal meningitis should be high on the list of differential diagnoses. Lumbar puncture should be performed early, even in the absence of fever, and consideration given to exploration and biopsy at the operative site for definitive diagnosis.

\section{REFERENCES}

1. Snider WD, Simpson DM, Nielsen S. Neurological complications of the acquired immune deficiency syndrome. Analysis of 50 patients. Ann Neurol 1983; 14: 403-418.
2. Parker JC, Jr., McCloskey JJ, Solanki KV. Candidosis - the most common post-mortem cerebral mycosis in an endemic fungal area. Surg Neurol 1976; 6: 123-128.

3. Lipton SA, Hickey WF, Morris JH, Loscalzo J. Candidal infection in the central nervous system. Am J Med 1984; 76:101-108.

4. Lyons RW, Andriole VT. Fungal infections of the CNS. Neurol Clin 1986; 4: 159-170.

5. Fleischmann J, Church JA, Lehrer RI. Case report; primary Candida meningitis and chronic granulomatous disease. Am J Med Sci 1986; 291: 334-341.

6. Gokhale YA, Ramnath RR, Patil RD, Prasar S, Kamble P, Balani S, Kelkar PN, Rammurthi K, Menon PS. Rhinocerebral mucormycosis associated with diabetic ketoacidosis. J Assoc Phys India 1991; 39: 970-971.

7. Walsh TJ, Hier DB, Caplan LR. Fungal infection of the central nervous system: comparative analysis of risk factors and clinical signs in 57 patients. Neurology 1985; 35: 1654-1657.

8. Peterson PK, Chao CC, Hu S, Thielen K, Shaskan EG. Gliobastoma, transforming growth factor - $\beta$, and Candida meningitis. Am J Med 1992; 92: 262-264.

9. Voice RA, Bradley SF, Sangeorzan JA, Kauffman CA. Chronic Candidal meningitis: an uncommon manifestation of candidiasis. Clin Infec Dis 1994; 19: 60-66.

10. Chesney, PJ, Teets, KC, Mulvihill, JJ, Salit, IE, Marks, MI. Successful treatment of Candida meningitis with amphotericin $B$ and 5-fluorocytosine in combination. J Pediatrics 1976; 89: 1017-1019 